\title{
Calcificação vascular na DRC
}

\author{
Vascular calcification in CKD
}

\section{Autores: \\ Maria Eugênia F. \\ Canziani \\ Rosa Maria A. Moysés}

1 A presença de calcificação vascular (CV) deve ser investigada em paciente com DRC (Opinião).

2 A avaliação da $\mathrm{CV}$ pode ser feita por métodos semiquantitativos ou quantitativos (Evidência).

3 A avaliação da CV deve ser feita anualmente (Opinião).

4 A prevenção da CV se faz por meio do controle dos seguintes fatores de risco modificáveis:

4.1 Fatores tradicionais: hipertensão arterial, diabetes melito, dislipidemia, obesidade, tabagismo e sedentarismo (Evidência).

4.2 Fatores não tradicionais: inflamação, estresse oxidativo, hiperfosfatemia, hipercalcemia, sobrecarga de $\mathrm{Ca}$ (consequente à ingestão e/ou solução de diálise), hipo e hiperparatireoidismo (Opinião).

\section{RACIONAL}

As doenças cardiovasculares (DCV) constituem a principal causa de mortalidade em pacientes com doença renal crônica (DRC). ${ }^{1} \mathrm{O}$ risco de óbito por DCV nessa população chega a ser 10 a 30 vezes maior que aquele observado na população geral. Esse aumento da mortalidade também ocorre em pacientes nos estágios precoces da DRC, ${ }^{2}$ assim como naqueles submetidos a transplante renal. ${ }^{3}$ A alta prevalência de DCV nos pacientes com DRC se deve, em parte, à elevada incidência de fatores de risco tradicionais para DCV nesta população. A presença de fatores de risco tradicionais, como diabetes, hipertensão, dislipidemia, idade avançada e sedentarismo, tem relação com o aparecimento e progressão da lesão aterosclerótica e sua calcificação, tanto na população geral como nos portadores de DRC. ${ }^{4}$

Entretanto, alguns estudos demonstraram que a ocorrência desses fatores não é suficiente para explicar a associação entre DCV e DRC. ${ }^{4}$ Fatores relacionados à uremia, como anemia, sobrecarga de volume, distúrbios do metabolismo mineral e ósseo, inflamação, aumento do estresse oxidativo, podem contribuir para o agravamento de lesões cardiovasculares preexistentes ou induzir o aparecimento das mesmas. ${ }^{5}$

Entre as complicações cardiovasculares da DRC, a CV tem recebido grande destaque na literatura médica na última década, o que se deve, essencialmente, ao fato de estar associada a um aumento de mortalidade. ${ }^{6,7} \mathrm{~A}$ CV é um achado frequente em pacientes submetidos à diálise ${ }^{8-10}$ e ocorre geralmente em dois locais distintos: nas camadas íntima e média dos vasos. Mais recentemente, estudos têm demonstrado a ocorrência de CV em pacientes na fase pré-dialítica. ${ }^{11}$

A calcificação localizada na íntima do vaso é considerada um marcador de aterosclerose, sendo sua extensão diretamente relacionada à gravidade da lesão aterosclerótica observada à angiografia. ${ }^{12} \mathrm{Na}$ última década, tem-se evidenciado o papel do processo inflamatório sobre a célula endotelial como elemento fundamental no desenvolvimento e na progressão das lesões ateroscleróticas. ${ }^{13}$ 
A CV localizada na camada média do vaso é extremamente prevalente em pacientes com DRC, e parece estar associada aos distúrbios do metabolismo mineral. ${ }^{14} \mathrm{Na}$ presença de distúrbios da remodelação óssea, como o aumento da reabsorção (alta remodelação) ou perda da capacidade-tampão do tecido ósseo (baixa remodelação), ocorre sobrecarga extracelular de Ca e P que favorece a calcificação extraóssea, principalmente a CV.

Vários estudos associaram o aumento das concentrações séricas de $\mathrm{Ca}$ e $\mathrm{P}$ com a $\mathrm{CV}$ em pacientes com DRC. ${ }^{15,16}$ Esse processo é ativo, semelhante à ossificação. Sabe-se que as células da musculatura vascular lisa sofrem uma transformação e adquirem características da célula osteoblástica, em que o fator de transcrição Cbfa1 parece ser a peçachave. ${ }^{17} \mathrm{O}$ transcription factors core binding factor $\alpha 1$ (Cbfa1) é um fator de transcrição, expressado por células precursoras mesenquimais da medula óssea, com a função de estimular a diferenciação dessas células em osteoblastos maduros. Essa célula osteoblasto-símile expressa várias proteínas características do tecido ósseo, como osteopontina, MGP (proteína Gla da matriz), fosfatase alcalina, osteocalcina e colágeno tipo $\mathrm{I}^{18}{ }^{18}$ Vários estudos demonstraram que o $\mathrm{P}$ é um dos fatores capazes de induzir a transformação da célula da musculatura lisa vascular, sendo que o $\mathrm{Ca}$ também contribui nesse processo, aumentando a expressão dos cotransportadores de $\mathrm{P}$, facilitando assim a entrada desse elemento na célula. ${ }^{19}$ Adicionalmente, toxinas presentes no soro urêmico, citocinas, radicais livres e glicose parecem acelerar a CV independentemente da concentração de $\mathrm{P} .{ }^{20}$

Vale ressaltar que uma parcela de pacientes, em torno de $20 \%$ na maioria dos estudos, não tem e não desenvolve $\mathrm{CV}$ ao longo do tempo. ${ }^{20}$ Esse dado sugere que algumas proteínas como MGP, fetuína-A, osteopontina e osteoprotegerina estejam envolvidas na inibição do processo de $\mathrm{CV}^{21-24}$

A ocorrência da CV tem sido associada a um aumento de mortalidade e confere valor prognóstico negativo independentemente de sua localização (na íntima ou na média). Em indivíduos com DRC, a calcificação da placa aterosclerótica parece ocorrer mais precocemente e de forma mais intensa do que na população geral, e sua progressão implica isquemia e fibrose miocárdica, que se manifestam como arritmias, insuficiência cardíaca e morte súbita. ${ }^{25}$

Estudos realizados já demonstraram que o grau de calcificação associa-se ao número de lesões diagnosticadas pela arteriografia. ${ }^{26}$ A calcificação da camada média das artérias leva a uma redução da complacência das grandes artérias de condução, o que resulta em aumento da pressão de pulso, barotrauma, redução da perfusão coronariana, disfunção vasomotora endotelial e hipertrofia ventricular esquerda. ${ }^{27}$

Os métodos de avaliação da CV podem ser:

\section{A. Qualitativo}

Radiografia simples: método de fácil obtenção e baixo custo, porém de baixa sensibilidade, que permite detectar a presença de $\mathrm{CV}$ em vasos dos diferentes segmentos do corpo.

\section{B. Semiouantitativo}

B.1 Radiografias de mãos e pelve - método simples e de baixo custo para avaliação da CV; consiste na divisão em quadrantes das radiografias das mãos e da pelve. Esse método mostrou uma correlação significativa com a calcificação coronariana em pacientes com DRC. A radiografia da pelve deve ser dividida em quadrantes por uma linha horizontal acima das cabeças dos fêmures e por uma linha vertical sobre a coluna vertebral. Já na radiografia das mãos, a linha vertical separa as duas mãos e a linha horizontal passa acima dos ossos do metacarpo. A presença de CV em cada quadrante é contada como um ponto, e sua ausência como zero. $\mathrm{O}$ escore final é a soma de todos os pontos que, portanto, variam de 0 a 8 . Só são atribuídos pontos para CV localizadas nas artérias ilíacas, femorais, radiais e digitais ${ }^{28}$.

B.2 Ultrassonografia arterial associada a radiografias - ultrassonografia de artérias carótidas, aorta abdominal, ileofemorais e poplíteas. A presença de calcificação é complementada pela realização de radiografias posteroanterior e perfil do abdômen, pelve e membros inferiores. A CV, em cada um desses locais, detectada na ultrassonografia ou no Rx, é contada como um ponto e sua ausência como zero. O escore final é a soma de todos os pontos, variando de 0 a $4 .^{29}$

\section{Quantitativo}

Tomografia coronariana computadorizada por feixe de elétrons ou tomografia computadorizada multisli$c e$ - técnicas que permitem a quantificação da $\mathrm{CV}$ e, quando realizadas em diferentes períodos, a análise da sua progressão. São realizadas em sincronia com ECG, o que permite a aquisição de imagens cardíacas no momento refratário do batimento cardíaco. O Ca presente na artéria coronária é quantificado utilizando-se o escore de Agatston, que é calculado pela multiplicação da área da placa por um coeficiente de densidade. 
A tomografia coronariana é um método não invasivo que permite a quantificação de $\mathrm{Ca}$ em cada segmento estudado. No entanto, esse método não possibilita a distinção entre calcificação da camada íntima e média. ${ }^{30}$

Além disso, outras medidas, como espessura do complexo íntima-média das carótidas, $\mathrm{CV}$ pelo ecocardiograma, CV avaliada em tomografia de tórax ou abdômen foram capazes de predizer o escore de $\mathrm{Ca}$ coronariano em pacientes com DRC. Adicionalmente, a velocidade da onda de pulso também se relaciona com a presença de calcificação coronariana; entretanto, esses dados são controversos. ${ }^{31}$

A recomendação de avaliação anual da calcificação baseou-se em alguns trabalhos que estudaram progressão de calcificação. Nesses estudos, a maioria realizada com pacientes no estágio $\mathrm{V} \mathrm{D}$, com seguimento de 1 a 3 anos, demonstraram que é possível detectar progressão da calcificação no período de 1 ano, utilizando métodos como radiografia, ultrassonografia de carótidas (medida da espessura do complexo íntima-média) e tomografias. ${ }^{31}$

A CV, uma vez presente, raramente regride. Portanto, o principal objetivo do tratamento deve ser a prevenção e a estabilização da CV já existente, através do controle dos fatores de risco modificáveis. Entre os fatores de risco tradicionais modificáveis, recomenda-se controle rigoroso dos níveis pressóricos, glicemia, lípides, abandono do tabagismo e realização de atividades físicas regulares. Desse modo, as medidas orientadas pela Sociedade Brasileira de Cardiologia para a prevenção da aterosclerose devem ser implementadas no cuidado dos pacientes com DRC, sendo que essa população deve ser considerada de alto risco já na fase pré-dialítica. ${ }^{32}$ Quanto aos fatores de risco não tradicionais, aqueles relacionados à uremia, uma atenção maior deve ser dada ao controle dos distúrbios do metabolismo mineral.

As concentrações séricas de $\mathrm{P}$, o produto CaxP e a sobrecarga de $\mathrm{Ca}$ correlacionam-se positivamente com a CV. Elevadas concentrações de Ca e P estão associadas ao aumento da mortalidade em pacientes com DRC. ${ }^{33}$ A CV tem sido relacionada à integridade do tecido ósseo. Assim, tanto as doenças ósseas de alta como de baixa remodelação, especialmente a DOA, ${ }^{34}$ têm sido associadas à CV. Adicionalmente, estudos epidemiológicos têm mostrado uma correlação inversa entre massa óssea e CV, tanto na população geral como nos pacientes com DRC. ${ }^{9,35}$ Desse modo, a prevenção da CV pressupõe um controle rigoroso das concentrações de $\mathrm{Ca}$ e $\mathrm{P}$, da sobrecarga de $\mathrm{Ca}$, assim como o controle da remodelação óssea.
Adicionalmente, medidas devem ser implementadas para minimizar a inflamação, como o controle rigoroso da água utilizada para diálise e a prevenção e tratamento de infecções. ${ }^{36}$ Portanto, a CV é um processo ativo de ossificação dos vasos, extremamente frequente na uremia, e que contribui para a elevada morbimortalidade cardiovascular presente nessa população.

Com relação ao impacto do tratamento do DMODRC sobre a progressão da CV, poucos estudos clínicos prospectivos e randomizados foram realizados, e os resultados foram conflitantes para alguns dos fatores de risco. O papel dos quelantes de $\mathrm{P}$ sobre a progressão da $\mathrm{CV}$ foi avaliado por cinco diferentes estudos clínicos, sendo que três deles identificaram benefício do uso de sevelamer sobre os sais de $\mathrm{Ca},{ }^{37-39}$ enquanto outros dois não obtiveram resultados semelhantes. ${ }^{40,41}$ Não há estudos que compararam o efeito da paratireoidectomia sobre a progressão da $\mathrm{CV}$, mas um estudo recentemente publicado mostrou benefício do uso de cinacalcete sobre a progressão da calcificação em pacientes portadores de hiperparatireoidismo secundário. ${ }^{42}$

Embora a dislipidemia seja considerada um fator de risco para CV, e estudos realizados na população sem DRC tenham demonstrado benefício do uso de estatinas sobre o número de eventos cardiovasculares, os mesmos achados não puderam ser confirmados nos pacientes portadores de DRC. De fato, os estudos $4 \mathrm{D}^{43}$ e AURORA ${ }^{44}$ não mostraram benefício do uso de estatinas no número de eventos nos pacientes em hemodiálise. Mais recentemente, os dados preliminares do estudo SHARP apontam para uma redução de eventos cardiovasculares em pacientes com DRC que utilizaram a combinação sinvastatina ezetimiba. Não há estudos que tenham avaliado o papel do uso de estatinas sobre a progressão da CV. A despeito disso, as recomendações atuais ainda são de tratar esses pacientes como de alto risco para DCV e manutenção de níveis adequados de colesterol e triglicérides. ${ }^{31,32}$

\section{REFERÊNCIAS}

1. Foley RN, Parfrey OS, Sarnak MJ. Clinical epidemiology of cardiovascular disease in chronic renal disease. Am J Kidney Dis 1998; 32:S112-S119.

2. Go AL, Chertow GM, Fan D et al. Chronic kidney disease and the risks of death, cardiovascular events and hospitalization. New Engl J Medicine 2004; 351:1296-305.

3. Collins AJ, Kasiske B, Herzog C et al. Excerpts from the United States Renal Data System 2004 annual data report: Atlas of end stage renal disease in the United States. Am J Kidney Dis 2005; 45(Suppl 1):A5-7, S1-28045. 
4. Longenecker JC, Coresh J, Powe NR et al. Traditional cardiovascular disease risk factors in dialysis patients compared with the general population: the CHOICE Study. J AM Soc Nephrol 2002; 13:1918-27.

5. Zoccali C. Cardiovascular risk in uraemic patients - is it fully explained by classical risk factors? Nephrol Dial Transplant 2000; 15:454-7.

6. Matsuoka M, Iseki K, Tamashiro M et al. Impact of high coronary artery calcification score (CACS) on survival in patients on chronic hemodialysis. Clin Exp Nephrol 2004; 8:54-8.

7. Ammirati AL, Dalboni MA, Cendoroglo $\mathrm{M}$ et al. The progression and impact of vascular calcification in peritoneal dialysis patients. Perit Dial Int 2007; 27:340-6.

8. Braun J, Oldendorf M, Moshage W et al. Electron beam computed tomography in the evaluation of cardiac calcification in chronic dialysis patients. Am J Kidney Dis 1996; 27:394-401.

9. Barreto DV, Barreto FC, Carvalho AB et al. Coronary calcification in hemodialysis patients: the contribution of traditional and uremia-related risk factors. Kidney Int 2005; 67:1576-82.

10. Ammirati AL, Dalboni MA, Cendoroglo $\mathrm{M}$ et al. Coronary artery calcification, systemic inflammation markers and mineral metabolism in a peritoneal dialysis population. Nephron Clin Pract 2006; 104:c33-40.

11. Tomiyama C, Higa A, Dalboni MA et al. The impact of traditional and non-traditional risk factors on coronary calcification in pre-dialysis patients. Nephrol Dial Transplant 2006; 21(9):2464-71.

12. Detrano R, Hsiai T, Wang S et al. Prognostic value of coronary calcification and angiographic stenoses in patients undergoing coronary angiography. J Am Coll Cardiol 1996; 27:285-90.

13. Burke AP, Tracy RP, Kolodgie F et al. Elevated C-reactive protein values and atherosclerosis in sudden coronary death: association with different pathologies. Circulation 2002; 105:2019-23.

14. Lomashvili KA, Cobbs S, Hennigar RA et al. Phosphate-induced vascular calcification: role of pyrophosphate and osteopontin. J Am Soc Nephrol 2004; 15:1392-401.

15. Goodman WG, Goldin J, Kuizon BD et al. Coronaryartery calcification in young adults with end-stage renal disease who are undergoing dialysis. N Engl J Med 2000; 342:1478-83.

16. Chertow GM, Raggi $\mathrm{P}$, Chasan-Taber $\mathrm{S}$ et al. Determinants of progressive vascular calcification in haemodialysis patients. Nephrol Dial Transplant 2004; 19:1489-96.

17. Jono S, McKee MD, Murry CE et al. Phosphate regulation of vascular smooth muscle cell calcification. Circ Res 2000; 87:E10-17.

18. Moe SM, O’Neill KD, Duan D et al. Medial artery calcification in ESRD patients is associated with deposition of bone matrix proteins. Kidney Int 2002; 61:638-47.

19. Yang H, Curinga G, Giachelli CM. Elevated extracellular calcium levels induce smooth muscle cell matrix mineralization in vitro. Kidney Int 2004; 66:2293-9.

20. Chen NX, Duan D, O'Neill KD et al. The mechanisms of uremic serum-induced expression of bone matrix proteins in bovine vascular smooth muscle cells. Kidney Int 2006;70:1046-53.
21. Luo G, Ducy P, McKee MD et al. Espontaneous calcification of arteries and cartilage in mice lacking matrix GLA protein. Nature 1997; 386:78-81.

22. Schafer C, Heiss A, Schwarz A et al. The serum protein alpha 2-Heremans-Schmid glycoprotein/fetuin-A is a systemically acting inhibitor of ectopic calcification. J Clin Invest 2003; 112:357-66.

23. Wada T, McKee MD, Steitz S. Calcification of vascular smooth muscle cell cultures: inhibition by osteopontin. Cir Res 1999; 84:166-78.

24. Bucay N, Sarosi I, Dunstan CR et al. Osteoprotegerin deficient mice develop early onset osteoporosis and arterial calcification. Genes Dev 1998; 12:1260-8.

25. McCullough PA. Cardiovascular care in end-stage renal disease. Adv Chronic Kidney Dis 2004; 11:245.

26. Haydar AA, Hujairi NM, Covic AA et al. Coronary artery calcification is related to coronary atherosclerosis in chronic renal disease patients: a study comparing EBCT-generated coronary artery calcium scores and coronary angiography. Nephrol Dial Transplant 2004; 19:2307-12.

27. Lemos MM, Jancikic AD, Sanches FM et al. Pulse wave velocity a useful tool for cardiovascular surveillance in pre-dialysis patients. Nephrol Dial Transplant 2007; 22:3527-32.

28. Adragao T, Pires A, Lucas C et al. A simple vascular calcification score predicts cardiovascular risk in haemodialysis patients. Nephrol Dial Transplant 2004; 19: 1480-8.

29. Blacher J, Guerin AP, Pannier B et al. Arterial calcifications, arterial stiffness, and cardiovascular risk in endstage renal disease. Hypertension 2001; 38:938-42.

30. Agatston AS, Janowitz WR, Hildner FJ et al. Quantification of coronary artery calcium using ultrafast computed tomography. J Am Coll Cardiol 1990; 15:827-32.

31. Diagnosis of CKD-MBD: vascular calcification. KDIGO Clinical practice guidelines for diagnosis, evaluation, prevention and treatment of chronic kidney disease mineral and bone disorder. Kidney International 2009; 76:S44-49.

32. Departamento de Aterosclerose da Sociedade Brasileira de Cardiologia. IV Diretriz Brasileira Sobre Dislipidemias e Prevenção da Aterosclerose. Arq Bras Cardiol 2007; 88:SI.

33. Block GA, Klassen PS, Lazarus JM et al. Mineral metabolism, mortality, and morbidity in maintenance hemodialysis. J Am Soc Nephrol 2004; 15:2208-18.

34. Safar ME, London GM, Plante GE. Arterial stiffness and kidney function. Hypertension 2004; 43:163-8.

35. Samelson EJ, Cupples LA, Broe KE et al. Vascular calcification in middle age and long-term risk of hip fracture: the Framingham study. J Bone Miner Res 2007; 22:1449-54.

36. Arizono K, Nomura K, Motoyama T et al. Use of ultrapure dialysate in reduction of chronic inflammation during hemodialysis. Blood Purif 2004; 22(suppl 2):26-9.

37. Chertow GM, Burke SK, Raggi P. Sevelamer attenuates the progression of coronary and aortic calcification in hemodialysis patients. Kidney Int 2002; 62:245-52.

38. Block GA, Spiegel DM, Ehrlich J et al. Effects of sevelamer and calcium on coronary artery calcification in patients new to hemodialysis. Kidney Int 2005; 68:1815-24. 
39. Russo D, Miranda I, Ruocco C et al. The progression of coronary artery calcification in predialysis patients on calcium carbonate or sevelamer. Kidney Int 2007; 72:1255-61.

40. Qunibi W, Moustafa M, Muenz LR et al. A 1-year randomized trial of calcium acetate versus sevelamer on progression of coronary artery calcification in hemodialysis patients with comparable lipid control: the Calcium Acetate Renagel Evaluation-2 (CARE-2) study. Am J Kidney Dis 2008; 51:952-65.

41. Barreto DV, Barreto $\mathrm{F}$ de $\mathrm{C}$, de Carvalho $\mathrm{AB}$ et al. Phosphate binder impact on bone remodeling and coronary calcification--results from the BRiC study. Nephron Clin Pract 2008; 110:c273-83.
42. Raggi P, Chertow GM, Torres PU et al. On behalf of the ADVANCE Study Group The ADVANCE study: a randomized study to evaluate the effects of cinacalcet plus low-dose vitamin D on vascular calcification in patients on hemodialysis. Nephrol Dial Transplant 2010 Dec 8. [Epub ahead of print].

43. Wanner C, Krane V, März W et al. German Diabetes and Dialysis Study Investigators. Atorvastatin in patients with type 2 diabetes mellitus undergoing hemodialysis. N Engl J Med 2005; 353(3):238-48.

44. Fellström BC, Jardine AG, Schmieder RE et al. AURORA Study Group. Rosuvastatin and cardiovascular events in patients undergoing hemodialysis. N Engl J Med 2009; 360(14):1395-407. 